\title{
Music Puzzle: An Audio-Based Computer Game That Inspires to Train Listening Abilities
}

\author{
Kjetil Falkenberg Hansen ${ }^{1}$, Rumi Hiraga ${ }^{2}$, Zheng Li $^{1}$, and Hua Wang ${ }^{1}$ \\ ${ }^{1}$ KTH Royal Institute of Technology \\ Lindstedtsvägen 24, 10044 Stockholm, Sweden \\ kjetil@kth.se \\ 2 Tsukuba University of Technology \\ 4-3-15, Amakubo, Tsukuba 305-8520, Japan
}

\begin{abstract}
The Music Puzzle is a computer game for tablets and smartphones using sounds for the gameplay. Just like an original picture is reconstructed from pieces with jigsaw puzzle, an original sound is reconstructed from musical segments with Music Puzzle. Each segment is distorted by shifting the pitch and equalization. To finish the game, the user listens to each segment visualized as pieces on the screen, reorders them, and corrects their pitch and equalization. The game has a possibility for deaf and hard of hearing people to improve their residual hearing ability since the observation shows their concentrating the game with sounds and preference for music.
\end{abstract}

Keywords: tablet game, hearing ability, audio based game, training.

\section{Introduction}

Our experiences tell that deaf and hard of hearing young people like to listen to music, and they enjoy using smartphones. Also, we are aware of the welldocumented beneficial effects of music [5, and that it is possible to train one's hearing to recover sensory abilities and thus music appreciation (e.g. [34]). Recently we conducted an experiment using an audio-based computer game called the "Music Puzzle", and preliminary results have been reported in [2]. The experiment showed positive results concerning how much time persons with hearing losses are willing to invest in playful hearing training, and that playing the game potentially can improve sound perception and thus appreciation of music.

In this paper we will briefly describe the gameplay and the experiment set-up and game settings. Also some of the more aesthetic considerations and choices will be discussed. For experiment results, see [2]. The novelty of the work is how we use music for gaming, and how we investigate differences between solving a task with music stimuli, speech stimuli or the combination of speech and music.

\section{The Music Puzzle Game}

The likeness to a traditional jigsaw puzzle game is clear as the task is to reconstruct a complete picture from smaller pieces, or in our case, a sound file from

D. Reidsma, H. Katayose, and A. Nijholt (Eds.): ACE 2013, LNCS 8253, pp. 540 543, 2013.

(C) Springer International Publishing Switzerland 2013 
distorted sound fragments. This was chosen for several reasons, but mainly it can be argued that a familiar game can be easier to get started with. Our experiment group, consisting of students with hearing losses, were not used to audio based games and therefore we chose to keep the task simple, but challenging.

In a jigsaw, the puzzle is the complete picture; traditionally a well-known image divided into many small pieces. We use a sound recording divided into several small sound segments. The user can start by listening to the full recording or even start without knowing the target sound; the latter could be compared to laying a puzzle without looking at the picture on the box. The puzzle thus becomes more of a riddle or secret message to be deciphered. The pieces of the jigsaw must be hooked together correctly in two dimensions with fitting forms, but in our case the pieces must be ordered temporally. To introduce a further challenge than just finding the correct order in one dimension, each piece is randomly changed in pitch and tone quality (hi-pass and lo-pass filtering). This makes it more complex than a jigsaw puzzle while keeping the concept.

To solve the puzzle the player has to listen very carefully to each sound fragment, choose correct audio filtering and reorder the pieces. This task has proved to be quite complex involving focussing on details in the sounds to get it right, which corresponded perfectly with our ambition of making a motivational hearing training game based on sounds, speech and music from everyday life.

The game was written for smartphones with Android 2.2 or above, and later including other Android devices. Audio programming was done in Pure Data (Pd) with the libpd library for mobile systems. $\mathrm{Pd}$ is a programming environment for audio and has the necessary functions for pitch shifting and filtering.

\subsection{Game Play}

The game provides a simple user interface with only a few visual objects in order for players to concentrate their efforts to listening to the sounds. First, the user sets the game mode by choosing a predetermined sound set (FirstFourth) as shown in Figure1(a), and the type of puzzle - speech, music, or mixed, Figure 1(b). These options were made for the experiments and can be changed to suit other purposes. After that, a ball appears in the center of the display, Figure1(c). When the user presses the ball, the target sound is played. The user is requested to shake the tablet to "break" the ball into several smaller pieces, as illustrated in Figure 1(d). The number of pieces that are created depends on both the force of the shaking and the chosen difficulty level.

The created pieces contain a segment of the original sound and have equal durations; in a typical scenario, a 15 seconds long puzzle (sound recording) is divided into six 2.5 seconds long pieces. The user can then press to listen to a piece, drag and reorder it. Since pitch and equalization shifts are applied to each piece, the user also has to correct the pitch and equalization for each piece individually. Correcting options appear when the user presses and holds a piece on the screen. Radio buttons for these options have random color and order each time the window opens to prevent giving visual cues to the player. Besides interacting with the sound pieces, users can use two cheat buttons for 


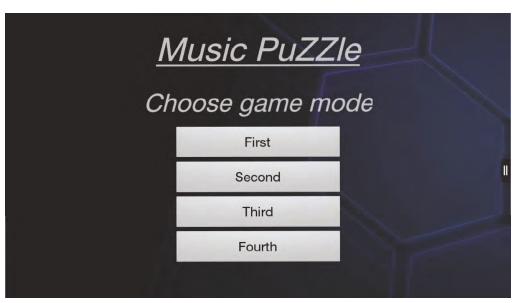

(a)

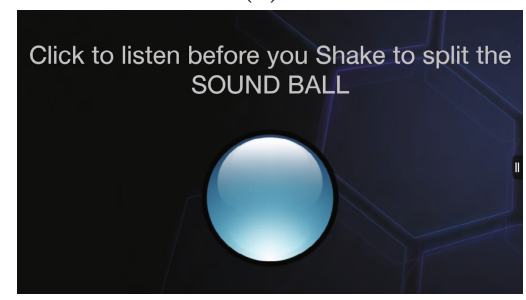

(c)

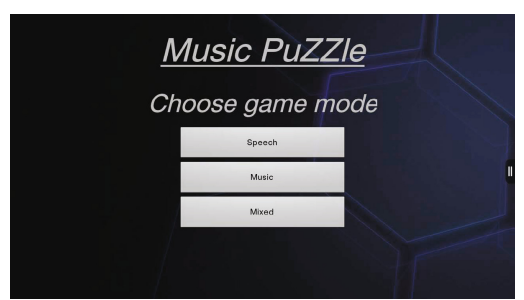

(b)

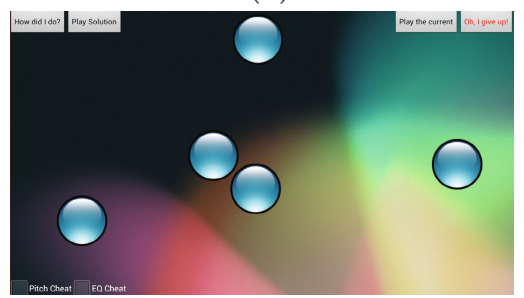

(d)

Fig. 1. The user interface. (a-b) The user sets the game mode by selecting between four sets of sounds and then between three puzzle types. (c) The user initiates the game by listening to the sound then shaking the tablet. (d) The game starts with the pieces randomly positioned on the screen.

automatically correcting pitch and filtering, found at the left bottom corner in Figure 1(d), and also use four buttons on the top row of the screen to evaluate a game session, play the original sound, play the current sequence as ordered on the screen, or dismiss the game.

\subsection{Difficulty Settings and Sounds}

The difficulty level is determined by several parameters: the length of the sound file, the number of pieces possible to create from shaking, the range of the pitch shift, the equalization's filtering settings, and not least the sound itself. These parameters impact greatly on each other in a complex way. For instance, a game consisting of more pieces is not always harder than a game with fewer pieces, and likewise a short sound file is not always harder than a longer one. Also, the change of pitch can make two pieces that in the original sounded differently appear very similar. Furthermore, symmetry effects caused by the division (for instance when dividing a 4-bar music segment into 2 or 4 pieces) or silent pieces (due to gaps in the original sound) file can make the task unexpectedly demanding.

Sounds are placed in folders, and settings are defined in a text file. Any sound recording can be used, depending on the experiment. In our experiments in [2] we have used read poetry, instrumental music and a combination of the reading and the music piece $(\mathrm{A}, \mathrm{B}, \mathrm{A}+\mathrm{B})$. In all, we had four sets of each which were not particularly different. In the earlier experiments [1] we used speech and music recordings of more varied character. Our experience from selecting the stimuli is 
that before dividing the sound, it is hard to know how difficult the task will be, and this demands that each game and sound should be tested for all the possible number of pieces. Sounds that seem overly easy, such as a read count-down of the numbers $5-4-3-2-1$, can still be sufficiently challenging.

\section{Summary of Preliminary Studies}

We have conducted several preliminary experiments 112 with hearing Swedish university students, and with Japanese university students with and without hearing losses. Most of them reported that they enjoyed playing the game. An interesting result was that most hearing participants preferred to play with speech, whereas most participants with hearing impairments showed a preference for music. Players without hearing loss perform overall slightly better than persons with hearing impairments, but less so than expected. The game seems to be challenging for all.

Further studies have been initiated, and we aim to reveal possible long-term training effects of using the game. Two other aims are to study how different sound stimuli are perceived and appreciated, and to see how difficulty settings (rates of pitch and filter changes, duration of pieces) can provide knowledge about sound perception in a playful way.

Acknowledgements. The authors want to thank the experiment participants, game testers and the paper reviewers for constructive feedback. This work is supported by a Grant-in-Aid for Scientific Research (C), the Japan Society for the Promotion of Science, and The Swedish Post and Telecom Authority (PTS).

\section{References}

1. Hansen, K.F., Li, Z., Wang, H.: A music puzzle game application for engaging in active listening. In: SIG Technical Reports: Proceedings of 97th Information Science and Music (SIGMUS) Research Conference, vol. (7), pp. 1-4 (electronic). Information Processing Society of Japan, Tokyo (2012)

2. Hiraga, R., Hansen, K.F.: Sound preferences of persons with hearing loss playing an audio-based computer game. In: Proceedings of the IMMPD and ACM Multimedia 2013, Barcelona, Spain. ACM Press (in press, 2013)

3. Kraus, N., Skoe, E., Parbery-Clark, A., Ashley, R.: Experience-induced malleability in neural encoding of pitch, timbre, and timing. Annals of the New York Academy of Sciences 1169, 543-557 (2009)

4. Särkämö, T., Pihko, E., Laitinen, S., Forsblom, A., Soinila, S., Mikkonen, M., Autti, T., Silvennoinen, H.M., Erkkil, J., Laine, M., Peretz, I., Hietanen, M., Tervaniemi, M.: Music and speech listening enhance the recovery of early sensory processing after stroke. Journal of Cognition and Neuroscience 22(12), 2716-2727 (2010)

5. UK Department for Education. Annex 3: The benefits of music (academic literature review). In: Department for Culture, Media and Sport (ed.) The Importance of Music. A National Plan for Music Education, pp. 42-44. Crown Copyright (2011) 